\title{
Healthy Preterm Newborn
}

National Cancer Institute

\section{Source}

National Cancer Institute. Healthy Preterm Newborn. NCI Thesaurus. Code C114931.

A healthy newborn whose only diagnoses are those related to prematurity. (NIAID) 